\title{
Reflection From a Sharply Bounded Ionosphere for VLF Propagation Perpendicular to the Magnetic Meridian
}

\author{
Douglass D. Crombie ${ }^{1}$

\begin{abstract}
Contribution From Central Radio Propagation Laboratory, National Bureau of Standards, Boulder, Colo.
\end{abstract}
(Received March 9, 1961; revised March 31, 1961)

\begin{abstract}
There is experimental evidence that VLF signals propagating from west to east suffer less attenuation than from east to west. Earlier work treating the case of nonreciprocal propagation along the magnetic equator is extended in latitude. The nonreciprocity shown by $\|R\|$ for highly oblique propagation along the magnetic equator persists when the reflection point moves towards a magnetic pole, but at the pole itself $\|R\|$ is reciprocal. To a first approximation $\perp R_{\perp}$ is reciprocal at all magnetic latitudes. The conversion coefficients ||$R \perp, \perp R \|$, are greater for east-to-west propagation than for propagation in the opposite direction, except at a magnetic pole where they are equal.
\end{abstract}

\section{Introduction}

There is now a considerable amount of experimental evidence [Crombie, 1958; Taylor 1960] showing that VLF propagation is nonreciprocal, since VLF signals received from a westerly quarter appear to suffer less attenuation than those received from the east. A qualitative explanation of such behavior in terms of interactions between the longitudinal movements of ionospheric electrons in the plane of incidence, and the transverse component of the earth's magnetic field was given by Crombie [1958].

Subsequently, Barber and Crombie [1959] calculated the reflection coefficient of a sharply bounded ionosphere and showed that this explanation was correct for the special case of waves polarized in the plane of incidence, propagating along the magnetic equator. This special case simplified the calculations since the magnetic field is horizontal and transverse to the plane of incidence.

It has also been shown [Crombie, 1960] that the nonreciprocity which could be expected from the lack of reciprocity exhibited by the reflection coefficient does occur when mode theory is used. Wait [1960, 1961] and Wait and Spies [1960], using some coefficients derived from the numerical data of Johler [1961], have shown that mode propagation depends on the direction of propagation. More recently, Dobrott and Ishimaru [1961] have also concluded that VLF propagation along the magnetic equator may be nonreciprocal.

It is the purpose of this paper to derive formulas for the reflection coefficient of a sharply bounded ionosphere in the rather more general case where plane waves are incident from the west, or from the east at any magnetic latitude.

\section{Introductory Theory}

The starting point of this work is a paper by Yabroff [1957a and b] in which, following an outline by Bremmer [1949], he derives perfectly general relationships between the fields in a sharply bounded ionosphere, and obtains the appropriate boundary equations. Yabroff considered the coordinate system shown in figure 1 . The vertical direction is $x$ and the earth's magnetic field $H_{e}$ is contained in the $x z$ plane. The angle of incidence of the waves is $\beta_{i}$, and the magnetic field of the earth is inclined to the vertical at an angle $\beta_{e}$. The incident, reflected and transmitted waves are described in the coordinate system $x^{\prime}, y^{\prime}, z^{\prime}$ in which for a wave

${ }^{1}$ Present address: Dominion Physical Laboratory, Lower Hutt, New Zealand. 


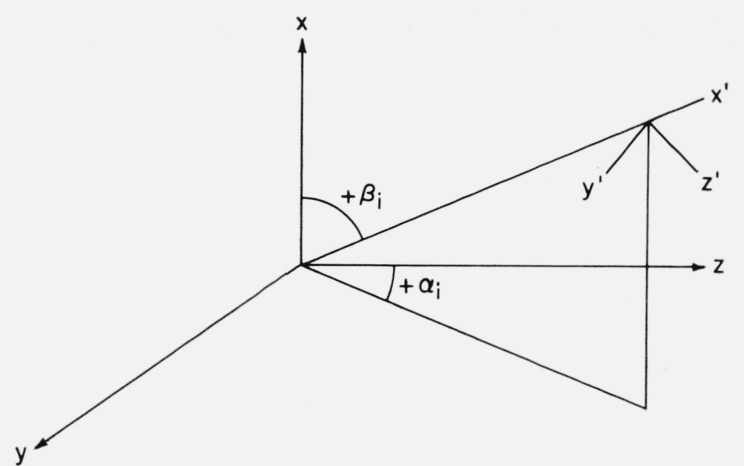

Figure 1. Coordinate system.

The positive values of $\boldsymbol{\beta}_{i}$ and $\alpha_{i}$ are measured clockwise from the positive $x$ and $z$ axes respectively.

propagating in the $\alpha_{i}, \beta_{i}$ direction, $z^{\prime}$ is in the $\alpha_{i}, \beta_{i}+90^{\circ}$ direction; $y^{\prime}$ is directed in the $\alpha_{i}+90^{\circ}$, $90^{\circ}$ direction and $x^{\prime}$ is in the direction $\alpha_{i}, \beta_{i}$. The lower boundary of the supposedly uniform ionosphere is the $x y$ plane. If $z$ is magnetic north, then $y$ is magnetic east.

The incident plane wave $F_{i}$ can be written as

$$
F_{i}=\operatorname{Re}\left\{F_{o i} \exp \left[j \frac{\omega}{c}\left(c t-x \cos \beta_{i}-y \sin \beta_{i} \sin \alpha_{i}-z \sin \beta_{i} \cos \alpha_{i}\right)\right]\right\}
$$

in which $\omega=$ wave angular frequency, $c$ is the velocity of light and $t$ is time. Since the boundary conditions must be satisfied it follows that the wave in the ionosphere $F_{m}$, arising from this incident wave, must be of the form

$$
F_{m}=\operatorname{Re}\left\{F_{o m} \exp \left[j \frac{\omega}{c}\left(c t-D x-y \sin \beta_{i} \sin \alpha_{i}-z \sin \beta_{i} \cos \alpha_{i}\right)\right]\right\}
$$

$D$ may be regarded as the cosine of the (complex) angle of refraction in the ionosphere.

Maxwell's equations and the equations of motion of an electron in a magnetic field can be written as

$$
\begin{aligned}
& \nabla \times \underset{\sim}{E}=-\mu_{0} \frac{\partial \underset{\sim}{H}}{\partial t} \\
& \left.\begin{array}{l}
\nabla \times \underset{\sim}{H}=-N e \underset{\sim}{V}+\epsilon_{0} \frac{\partial \underset{\sim}{E}}{\partial t} \\
m \frac{d v}{d t}=-e \underset{\sim}{E}-m \nu \underset{\sim}{V}-\mu_{0}\left(\underset{\sim}{V} \times \underset{\sim}{H_{e}}\right)
\end{array}\right\}
\end{aligned}
$$

in which, $-e, m$ are the charge and mass of an electron,

$\underset{\sim}{V}$ is the electron velocity,

$\tilde{\nu}$ is the collision frequency,

$\epsilon_{0}, \mu_{0}$ are the permittivity and permeability of free space.

From these three equations the three components of $\underset{\sim}{H}$ and $\underset{\sim}{V}$ may be eliminated giving three equations relating the components of $E$. In matrix form these equations are:

$$
\left[\begin{array}{ccc}
1-a^{2}-\frac{s^{2}-h_{L}^{2}}{s\left(s^{2}-h^{2}\right)} & a_{T} D-\frac{j h_{T}}{s^{2}-h^{2}} & a_{L} D+\frac{h_{T} h_{L}}{s\left(s^{2}-h^{2}\right)} \\
a_{T} D+\frac{j h_{T}}{s^{2}-h^{2}} & 1-a_{L}^{2}-D^{2}-\frac{s}{s^{2}-h^{2}} & a_{L} a_{T}-\frac{j h_{L}}{s^{2}-h^{2}} \\
a_{L} D+\frac{h_{T} h_{L}}{s\left(s^{2}-h^{2}\right)} & a_{T} a_{L}+\frac{j h_{L}}{s^{2}-h^{2}} & 1-D^{2}-a_{T}^{2}-\frac{s^{2}-h_{T}^{2}}{s\left(s^{2}-h^{2}\right)}
\end{array}\right]\left[\begin{array}{c}
E_{x} \\
E_{y} \\
E_{z}
\end{array}\right]=0
$$


wh ere

$$
\begin{aligned}
& s=(1-j Z) / X \quad h=Y / X, h_{L}=h \cos \beta_{e}, h_{T}=h \sin \beta_{e} \\
& X=N e^{2} / m \epsilon_{0} \omega^{2}={\text { (normalized plasma frequency })^{2} .} \\
& Y=\mu_{0} e H_{e} / \omega m=\text { normalized gyro frequency. } \\
& Z=\nu / \omega=\text { normalized collision frequency. } \\
& a_{L}=\sin \beta_{i} \cos \alpha_{i}, a_{T}=\sin \beta_{i} \sin \alpha_{i}, a=\sin \beta_{i} .
\end{aligned}
$$

In order that $\underset{\sim}{E}$ be finite the determinant of (2) must be zero. Thus

where

$$
b_{4} D^{4}+b_{3} D^{3}+b_{2} D^{2}+b_{1} D+b_{0}=0
$$

$$
\begin{aligned}
& b_{4}=s\left(s^{2}-h^{2}\right)-s^{2}+h_{L}^{2} \\
& b_{3}=2 a_{L} h_{L} h_{T} \\
& b_{2}=2 s\left[\left(1-a^{2}\right) h^{2}-(s-1)\left(s-a^{2} s-1\right)\right]-h_{T}^{2}\left(1-a_{L}^{2}\right)-\left(2-a^{2}\right) h_{L}^{2} \\
& b_{1}=-2\left(1-a^{2}\right) a_{L} h_{L} h_{T} \\
& b_{0}=(s-1)\left\{\left[\left(1-a^{2}\right) s-1\right]^{2}-\left(1-a^{2}\right)^{2} h_{T}^{2} \cos ^{2} \alpha_{i}\right\} \\
& \qquad \quad-\left(1-a^{2}\right)\left[\left(1-a^{2}\right) s-1\right]\left(h_{T}^{2} \sin ^{2} \alpha_{i}+h_{L}^{2}\right) .
\end{aligned}
$$

The four complex values of $D$ obtained from this quartic represent the two upgoing and two downgoing characteristic waves which can propagate independently in the medium. Values of $D$ with positive real and negative imaginary parts represent upgoing waves.

\subsection{Boundary Equations}

Since the total tangential fields $E_{y}, E_{z}, H_{y}$, and $H_{z}$ must be continuous across the boundary, the following boundary equations are obtained:

From $E_{y}$ :

$$
E_{y^{\prime} i} \cos \alpha_{i}+E_{z^{\prime} i} \cos \beta_{i} \sin \alpha_{i}+E_{y^{\prime} r} \cos \alpha_{i}-E_{z^{\prime} r} \cos \beta_{i} \sin \alpha_{i}=P_{1} E_{z 1}+P_{2} E_{z 2}
$$

From $E_{z}$ :

$$
-E_{y^{\prime} i} \sin \alpha_{i}+E_{z^{\prime} i} \cos \beta_{i} \cos \alpha_{i}-E_{y^{\prime} r} \sin \alpha_{i}-E_{z^{\prime} r} \cos \beta_{i} \cos \alpha_{i}=E_{z 1}+E_{z 2}
$$

From $H_{y}$ :

$$
E_{y^{\prime} i} \cos \beta_{i} \sin \alpha_{i}-E_{z^{\prime} i} \cos \alpha_{i}-E_{y^{\prime} r} \cos \beta_{i} \sin \alpha_{i}-E_{z^{\prime} r} \cos \alpha_{i}=S_{1} E_{z 1}+S_{2} E_{z 2}
$$

From $H_{z}$ :

$$
E_{y^{\prime} i} \cos \beta_{i} \cos \alpha_{i}+E_{z^{\prime} i} \sin \alpha_{i}-E_{y^{\prime} r} \cos \beta_{i} \cos \alpha_{i}+E_{z^{\prime} r} \sin \alpha_{i}=T_{1} E_{z 1}+T_{2} E_{z 2}
$$

In these equations

$$
\begin{aligned}
& P=E_{y} / E_{z}=M^{-1}\left\{-\left[1-a^{2}-\frac{s^{2}-h_{L}^{2}}{s\left(s^{2}-h^{2}\right)}\right]\left[a_{L} a_{T}-\frac{j h_{L}}{s^{2}-h^{2}}\right]+\left[a_{T} D+\frac{j h_{T}}{s^{2}-h^{2}}\right]\left[a_{L} D+\frac{h_{L} h_{T}}{s\left(s^{2}-h^{2}\right)}\right]\right\} \\
& Q=E_{x} / E_{z}=M^{-1}\left\{-\left[a_{L} D+\frac{h_{T} h_{L}}{s\left(s^{2}-h^{2}\right)}\right]\left[1-a_{L}^{2}-D^{2}-\frac{s}{s^{2}-h^{2}}\right]+\left[a_{L} a_{T}-\frac{j h_{L}}{s^{2}-h^{2}}\right]\left[a_{T} D-\frac{j h_{T}}{s^{2}-h^{2}}\right]\right\}
\end{aligned}
$$


where $M$ is given by

and

$$
M=\left[1-a^{2}-\frac{s^{2}-h_{L}^{2}}{s\left(s^{2}-h^{2}\right)}\right]\left[1-a_{L}^{2}-D^{2}-\frac{s}{s^{2}-h^{2}}\right]-\left[a_{T} D-\frac{j h_{T}}{s^{2}-h^{2}}\right]\left[a_{T} D+\frac{j h_{T}}{s^{2}-h^{2}}\right]
$$

$$
\begin{gathered}
R=\eta_{0} H_{x} / E_{z}=a_{T}-a_{L} P \\
S=\eta_{0} H_{y} / E_{z}=a_{L} Q-D \\
T=\eta_{0} H_{z} / E_{z}=D P-a_{T} Q .
\end{gathered}
$$

In which

$$
\eta_{0}=\sqrt{\mu_{0} / \epsilon_{0}}
$$

\subsection{Reflection Coefficient $R$}

This can be written in matrix form as

$$
R=\left[\begin{array}{l}
\| R_{\| \perp} R_{\|} \\
R_{\perp} R_{\perp} R_{\perp}
\end{array}\right] .
$$

The first subscript denotes whether the electric field is parallel $(\|)$ or pendicular $(\perp)$ to the plane of incidence, and the second subscript refers in the same way to the electric field of the reflected wave. The components of $R$ are defined as follows:

$$
\begin{array}{lll}
{ }_{\|} R_{\|}=E_{z^{\prime} \tau} / E_{z^{\prime} i} & (10 \mathrm{a}) . & { }_{\perp} R_{\|}=E_{z^{\prime} \tau} / E_{y^{\prime} i} . \\
{ }_{\|} R_{\perp}=E_{y^{\prime} r} / E_{z^{\prime} i} & (10 \mathrm{~b}) . & { }_{\perp} R_{\perp}=E_{y^{\prime} \tau} / E_{y^{\prime} i} .
\end{array}
$$

The components of $R$ are obtained by solution of the boundary eqs (4) for the appropriate pairs of electric fields.

\section{Application to Propagation Along a Line of Magnetic Latitude}

The work of Yabroff given above is perfectly general and expressions for the reflection coefficient could be derived for any direction of propagation, and for any orientation of the magnetic field. The labor involved would be excessive, however, and the resulting expressions cumbersome. The more practical approach to this general case is to use a computer for the solution of the boundary eqs (4). This has been done by Johler and Walters [1960] for propagation in the magnetic meridian. Johler [1961] has also calculated numerically, reflection coefficients for propagation at angles of $0^{\circ}, 60^{\circ}, 120^{\circ}, 180^{\circ}, 240^{\circ}, 300^{\circ}$ to the magnetic meridian, omitting the directions $90^{\circ}$ and $270^{\circ}$, with which this paper is concerned.

However, in the case of propagation along a line of magnetic latitude the expressions obtained for the reflection coefficient are relatively compact as will be shown below, although even in this case the algebra is very tedious.

For magnetic east-to-west or west-to-east propagation $\alpha_{i}=90^{\circ}$ or $270^{\circ}$. Thus, $a_{L}=0$. Making this substitution in eqs (4) gives for the boundary equations:

$$
\begin{aligned}
& E y: E_{z}{ }_{i} C-E_{z}{ }_{r} C=P_{1} E_{z 1}+P_{2} E_{z 2} \\
& H z: E_{z}{ }^{\prime}{ }+E_{z}{ }_{r}{ }_{r}=T_{1} E_{z 1}+T_{2} E_{z 2} \\
& E z:-E_{y}{ }^{\prime}{ }_{i}-E_{y}{ }_{r}{ }_{r}=E_{z 1}+E_{z 2} \\
& H y: E_{y}{ }_{i} C-E_{y}{ }_{r} C=S_{1} E_{z 1}+S_{2} E_{z 2}
\end{aligned}
$$

where $C=\cos \beta_{i}$.

(8b) and (8c) reduce to $T=D P-a_{T} Q$

$$
S=-D
$$


while (5), (6), and (7) reduce to

$$
\begin{gathered}
P=P^{\prime} M^{-1}=M^{-1}\left\{j\left[1-a^{2}-\frac{s^{2}-h_{L}^{2}}{s\left(s^{2}-h^{2}\right)}\right]+\frac{h_{T}}{s}\left(a_{T} D+\frac{j h_{T}}{s^{2}-H^{2}}\right)\right\} \frac{h_{L}}{s^{2}-h^{2}} \\
Q=Q^{\prime} M^{-1}=M^{-1}\left\{-j\left(a_{T} D-\frac{j h_{T}}{s^{2}-h^{2}}\right)-\frac{h_{T}}{s}\left(1-D^{2}-\frac{s}{s^{2}-h^{2}}\right)\right\} \frac{h_{L}}{s^{2}-h^{2}} \\
M=\left[1-a^{2}-\frac{s^{2}-h_{L}^{2}}{s\left(s^{2}-h^{2}\right)}\right]\left[1-D^{2}-\frac{s}{s^{2}-h^{2}}\right]-\left[a_{T} D^{2}+\frac{h_{T}^{2}}{\left(s^{2}-h^{2}\right)^{2}}\right]
\end{gathered}
$$

The quantities $M, P, Q, S, T$, carry the same subscripts as the $D$ which they contain.

\subsection{Solution of Boundary Equations}

The boundary eqs (11) can be solved in the following way for the components of $R$. ${ }_{\|} R_{\|}$ is taken as an example. From (11a) and (11b)

$$
{ }_{\|} R_{\|}=E_{z}{ }^{\prime} / E_{z}{ }^{\prime}{ }_{i}=\frac{T_{1} C-P_{1}+\left(E_{z 2} / E_{z 1}\right)\left(T_{2} C-P_{2}\right)}{T_{1} C+P_{1}+\left(E_{z 2} / E_{z 1}\right)\left(T_{2} C+P_{2}\right)} .
$$

From (11c) and (11d), on putting $E_{y}{ }_{i}=0$ it follows that

$$
E_{z 2} / E_{z 1}=-\left(C+D_{1}\right) /\left(C+D_{2}\right) \text {. }
$$

Substitution of (18) in (17) gives

Similarly

$$
\begin{gathered}
{ }_{\|} R_{\|}=\Delta^{-1}\left[\left(T_{1} C-P_{1}\right)\left(C+D_{2}\right)-\left(T_{2} C-P_{2}\right)\left(C+D_{1}\right)\right], \\
\Delta=\left(T_{1} C+P_{1}\right)\left(C+D_{2}\right)-\left(T_{2} C+P_{2}\right)\left(C+D_{1}\right) .
\end{gathered}
$$

Substitution for $T_{1}, P_{1}, T_{2}$ and $P_{2}$ in these equations gives, after much work, the following relatively compact expressions for $\| R_{\|}$and ${ }_{\perp} R_{\perp}$

$$
\begin{aligned}
\| R_{\|}= & \Delta_{1}^{-1} \llbracket\left\{j\left[\left(1-\frac{1}{s}\right) C D_{1} D_{2}-D_{0}^{2}\left(C+D_{1}+D_{2}\right)\right]+\frac{h_{T} a_{T}}{s}\left[C\left(C+D_{1}+D_{2}\right)-D_{1} D_{2}\right]\right\}\left[\frac{s^{2}-h_{L}^{2}}{s\left(s^{2}-h^{2}\right)}\right. \\
& -1]\left(D_{1}+D_{2}\right)+\left[j\left(2 D_{0}^{2}+\frac{a^{2}}{s}\right)-\frac{2 C a_{T} h_{T}}{s}\right]\left[\left(\frac{s}{s^{2}-h^{2}}-1\right)\left(D_{0}^{2}+D_{1} D_{2}\right)+\frac{h_{T}^{2}-h_{L}^{2} D_{1} D_{2}}{s\left(s^{2}-h^{2}\right)}\right] \rrbracket \\
{ }_{\perp} R_{\perp}= & \Delta_{1}^{-1} \llbracket\left\{\{ j [ D _ { 0 } ^ { 2 } ( C - D _ { 1 } - D _ { 2 } ) - D _ { 1 } D _ { 2 } ( 1 - \frac { 1 } { s } ) C ] - \frac { h _ { T } a _ { T } } { s } [ D _ { 1 } D _ { 2 } - C ( C + D _ { 1 } + D _ { 2 } ) ] \} \left[\frac{s^{2}-h_{L}^{2}}{s\left(s^{2}-h^{2}\right)}\right.\right. \\
& -1]\left(D_{1}+D_{2}\right)+\left[j\left(2 D_{0}^{2}+\frac{a^{2}}{s}\right)+\frac{2 C h_{T} a_{T}}{s}\right]\left[\left(\frac{s}{s^{2}-h^{2}}-1\right)\left(D_{0}^{2}+D_{1} D_{2}\right)+\frac{h_{T}^{2}-h_{L}^{2} D_{1} D_{2}}{s\left(s^{2}-h^{2}\right)}\right] \rrbracket,
\end{aligned}
$$

in which

$$
\begin{aligned}
\Delta_{1}=\Delta\left[\frac{h_{L}}{s^{2}-h^{2}}\left(D_{1}-D_{2}\right)\right]=\left\{j\left[\left(1-\frac{1}{s}\right) C D_{1} D_{2}+D_{0}^{2}\left(C+D_{1}+D_{2}\right)\right]\right. \\
\left.+\frac{h_{T} a_{T}}{s}\left[C\left(C+D_{1}+D_{2}\right)+D_{1} D_{2}\right]\right\}\left[\frac{s^{2}-h_{L}^{2}}{s\left(s^{2}-h^{2}\right)}-1\right]\left(D_{1}+D_{2}\right) \\
+j \frac{a^{2}}{s}\left[\left(\frac{s}{s^{2}-h^{2}}-1\right)\left(D_{0}^{2}+D_{1} D_{2}\right)+\frac{h_{T}^{2}-h_{L}^{2} D_{1} D_{2}}{s\left(s^{2}-h^{2}\right)}\right]
\end{aligned}
$$


and $D_{0}^{2}=1-a^{2}-1 / s$, the value of $D^{2}$ when there is no magnetic field present.

Equation (22) reduces to

$$
{ }_{\perp} R_{\|}=-\Delta_{1}^{-1} \frac{2 h_{L}}{s^{2}-h^{2}}\left[\left(1-\frac{1}{s}\right) D_{0}^{2}+\frac{h_{T}^{2} a_{T}^{2}}{s^{2}}\right] C .
$$

Equation (21) reduces to

$$
{ }_{\|} R_{\perp}=\Delta_{1}^{-1} \cdot 2 C M_{1} M_{2}\left(\frac{s^{2}-h_{L}^{2}}{h_{L}}\right) .
$$

When $M_{1} M_{2}$ is expanded, it is found to contain terms involving $D_{1}^{2}+D_{2}^{2}$ and $D_{1}^{2} D_{2}^{2}$. These are respectively equal to $-b_{2} / b_{0}$ and $b_{4} / b_{0}$ in eq (3). Substitution of these in (28) gives, after considerable reduction,

$$
{ }_{\|} R_{\perp}=-\Delta_{1}^{-1} \frac{2 h_{L}}{s^{2}-h^{2}}\left[\left(1-\frac{1}{s}\right) D_{0}^{2}+\frac{h_{T}^{2} a_{T}^{2}}{s^{2}}\right] C
$$

which is identical to ${ }_{\perp} R_{\|}$.

\section{Discussion of Reflection Coefficient Formulas}

These formulas have been checked under various limiting conditions to ensure that they conform with other published results.

\subsection{No Magnetic Field}

If the magnetic field is zero then $\|_{\|}$and ${ }_{\perp} R_{\perp}$ reduce to

$$
\begin{aligned}
{ }_{\|} R_{\|} & =\frac{\left(D_{0}^{2}+a^{2}\right) C-D_{0}}{\left(D_{0}^{2}+a^{2}\right) C+D_{0}} \\
{ }_{\perp} R_{\perp} & =\frac{C-D_{0}}{C+D_{0}}
\end{aligned}
$$

while ${ }_{\|} R_{\perp}={ }_{\perp} R_{\|}=0$. These limiting forms were given by Yabroff [1959b].

\subsection{Horizontal Magnetic Field}

If the magnetic field is horizontal, $h_{L}=0, h_{T}=h$, and (19) reduces to

$$
{ }_{\|} R_{\|}=\frac{\left[j D_{2}\left(1-\frac{1}{s}\right)+\frac{h_{T} a_{T}}{s}\right] C-\left(j D_{1}^{2}+\frac{h_{T} a_{T} D_{2}}{s}\right)}{\left[j D_{2}\left(1-\frac{1}{s}\right)+\frac{h_{T} a_{T}}{s}\right] C+\left(j D_{1}^{2}+\frac{h_{T} a_{T} D_{2}}{s}\right)} .
$$

From figure $1, h_{T}$ is negative and for west-to-east propagation $a_{T}$ is also negative. In Barber and Crombie's paper [1959] their term equivalent to $h_{T} a_{T}$ was taken as negative for west-toeast propagation, because of the different coordinate systems used by them. Bearing this in mind it is possible to show that (30) is identical with their earlier result.

If in $(25) h_{L}=0$ then $(25)$ reduces again to

$$
{ }_{\perp} R_{\perp}=\frac{C-D_{1}}{C+D_{1}}=\frac{C-D_{0}}{C+D_{0}}
$$

confirming the result of Barber and Crombie [1959]. It is easily seen from (27) and (29) that when $h_{L}=0$ both ${ }_{\perp} R_{\|}$and ${ }_{\|} R_{\perp}$ become zero. 


\subsection{Vertical Incidence, Vertical Magnetic Field}

Here $h_{T}, a_{T}, a$ become zero and $C=1$.

Substitution in (24), (25), (27), (29), give

$$
{ }_{\|} R_{\|}={ }_{\perp} R_{\perp}=\frac{D_{1} D_{2}-1}{\left(D_{1}+1\right)\left(D_{2}+1\right)},{ }_{\perp} R_{\|}={ }_{\|} R_{\perp}=j \frac{D_{1}-D_{2}}{\left(D_{1}+1\right)\left(D_{2}+1\right)}
$$

which are results previously obtained by Bremmer [1949].

\subsection{Reciprocity}

The expressions (24), (25), (27), and (29) all contain $h_{T} a_{T}$. This is positive for west-toeast propagation and negative for east-to-west propagation. Thus, provided that $h_{T}$ is not zero, all the reflection coefficient terms may exhibit nonreciprocity.

\section{Numerical Work}

In order to evaluate the expressions for the reflection coefficients it is necessary first of all to find $D_{1}$ and $D_{2}$ from (3). For propagation along a line of magnetic latitude $a_{L}=0$ and (3) becomes a quadratic in $D^{2}$. Thus

$$
b_{4} D^{4}+b_{2} D^{2}+b_{0}=0,
$$

and

$$
D^{2}=\left[-b_{2} \pm \sqrt{b_{2}^{2}-4 b_{0} b_{4}}\right]\left(2 b_{4}\right)^{-1} .
$$

Substituting $a_{L}=0$ into eqs (3a) gives

$$
\begin{aligned}
b_{2} & =2 s\left[\left(1-a^{2}\right) h^{2}-s(s-1) D_{0}^{2}\right]-h_{T}^{2}-\left(2-a^{2}\right) h_{L}^{2} \\
b_{1}^{2}-4 b_{0} b_{4} & =h^{4}-2\left(1-a^{2}\right) h^{2} h_{L}^{2}+4 s(s-1) D_{0}^{2} h_{L}^{2}+\left(1-a^{2}\right)^{2} h_{L}^{4} \\
b_{4} & =s\left(s^{2}-h^{2}\right)-\left(s^{2}-h_{L}^{2}\right) .
\end{aligned}
$$

The values of $D_{1}, D_{2}$ having positive real and negative imaginary parts are chosen since these represent the upgoing "ordinary" and "extraordinary" waves in the ionosphere.

Some special cases are of interest in obtaining the results of section 4 .

For $h=0 ; D_{1}^{2}=D_{2}^{2}=D_{0}^{2}=1-a^{2}-1 / s$.

For $h_{L}=0 ; h_{T}=h$ and $D_{1}^{2}=D_{0}^{2}, \quad D_{2}^{2}=1-a^{2}-\frac{s-1}{s^{2}-h^{2}-s}$.

For $h_{T}=0 ; h_{L}=h$ and $a=0, \quad D_{1,2}^{2}=1-\frac{1}{s \pm h}$.

Some approximate calculations have been made for daytime conditions to show the effect of the angle of dip of the earth's magnetic field on the four reflection coefficient terms using the formulas (24), (25), (27), and (29) developed above. The calculations are based on the following parameters:

$$
\begin{aligned}
& \omega_{0}=10^{6} \text { corresponding to an electron density } \approx 310 / c c, \\
& \nu=10^{7} / \mathrm{sec}, \omega=10^{5} / \mathrm{sec}, \alpha_{i} \approx 78^{\circ} 80^{\prime}, C=0.2 .
\end{aligned}
$$

The earth's magnetic field has been taken as 0.5 gauss and the inclination of the field to the vertical $\beta_{e}=90^{\circ}, 60^{\circ}, 30^{\circ}$, and $0^{\circ}$. The results of these calculations are shown in table 1 . 


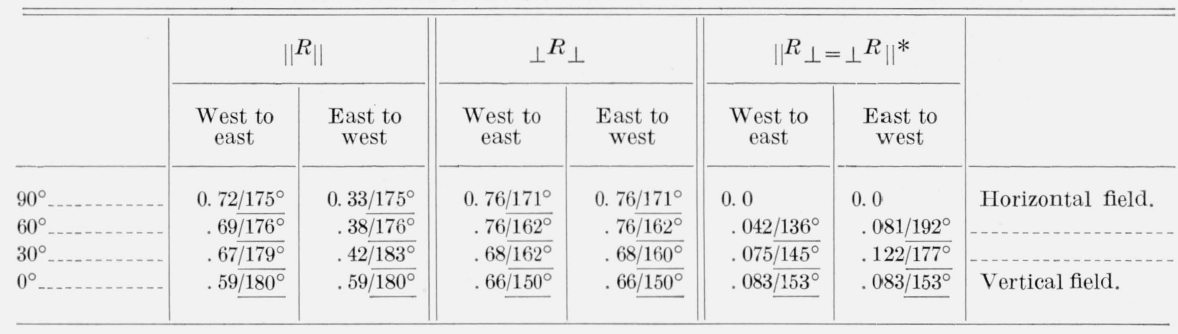

${ }^{*}$ For propagation in the Northern Hemisphere the phase angle of $\|_{\perp} R_{\perp}$ and $R_{\|}$should be reduced by $180^{\circ}$.

It is clearly seen from this table that ${ }_{\|} R_{\|,\|} R_{\perp}$ and ${ }_{\perp} R_{\|}$all show nonreciprocal properties, whereas ${ }_{\perp} R_{\perp}$ does not. In the case of ${ }_{\|} R_{\|}$the difference between east-to-west and west-to-east propagation is greatest at the magnetic equator where the magnetic field is horizontal, and gradually decreases as the reflection point moves nearer a magnetic pole. Nevertheless, the difference is very appreciable even when the angle of dip is $60^{\circ}\left(\beta_{e}=30^{\circ}\right)$. At the pole, where the magnetic field is vertical the two reflection coefficients are equal, since there is then no transverse field.

At the magnetic equator ${ }_{\|} R_{\perp}$ and ${ }_{\perp} R_{\|}$are zero. When the magnetic field is inclined $R_{\perp}$ and ${ }_{\perp} R_{\|}$become finite, but again their values for east-to-west and west-to-east propagation differ. This difference again disappears at the magnetic pole.

Despite the fact that eq (25) for ${ }_{\perp} R_{\perp}$ contains $a_{T} h_{T}$, it seems from table 1 that, for the values of the parameters used, ${ }_{\perp} R_{\perp}$ is essentially reciprocal. Thus in (25) the variations due to the sign of $a_{T} h_{T}$ must, to a first approximation cancel. This presumably will not necessarily occur for other values of the parameters.

The above numerical calculations have been made for highly oblique incidence, since long distance VLF propagation is of interest at present. However, the formulas are applicable at any angle of incidence. Thus the predictions of the formulas could be tested by making observations of ${ }_{\|} R_{\|}$and/or ${ }_{\|} R_{\perp}$ at points situated at equal distances to the west and east of a VLF transmitter. Alternatively, observation of the ground-interference pattern to the west and east of the transmitter could be made with airborne equipment.

\section{Conclusions}

This paper extends the earlier work of Barber and Crombie [1959] to the case of west-toeast and east-to-west propagation at any magnetic latitude. The nonreciprocity shown by ${ }_{\|} R_{\|}$for highly oblique propagation along the magnetic equator persists when the reflection point moves towards a magnetic pole, but at the pole itself ${ }_{\|} R_{\|}$is reciprocal. To a first approximation at least, ${ }_{\perp} R_{\perp}$ is reciprocal at all magnetic latitudes. However, the conversion coefficients $\| R_{\perp},{ }_{\perp} R_{\|}$, which are shown to be equal, are greater for east-to-west propagation than for propagation in the opposite direction, except at a magnetic pole where they are equal.

The work described in this paper was done while the writer was on a short visit to the Boulder Laboratories of the National Bureau of Standards. The writer wishes to express his appreciation for this opportunity, particularly to Dr. J. R. Wait through whom it was arranged.

\section{References}

Barber, N. F., and D. D. Crombie, V.L.F. reflections from the ionosphere in the presence of a transverse magnetic field, J.A.T.P. 16, 37 (1959).

Bremmer, H., Terrestrial Radio Waves, ch. 11 (Elsevier Publishing Co., New York, N.Y., 1949).

Crombie, D. D., Differences between east-west and west-east propagation of V.L.F. signals over long distances, J.A.T.P. 12, 110 (1958).

Crombie, D. D., On the mode theory of VLF propagation in the presence of a transverse magnetic field, J. Research NBS 64D (Radio Prop.), No. 3, 265-267 (May-June 1960). 
Dobrott, D., and A. Ishimaru, East-west effect on VLF mode transmission across the earth's magnetic field, J. Research NBS 65D (Radio Prop.) No. 1, 47-52 (Jan.-Feb. 1961).

Johler, J. Ralph, Magneto-ionic propagation phenomena in low- and very-low-radiofrequency waves reflected by the ionosphere, J. Research NBS 65D (Radio Prop.) No. 1, 53-65 (Jan.-Feb. 1961).

Johler, J. R., and L. C. Walters, On the theory of reflection of low and very low frequencies from the ionosphere, J. Research NBS 64D (Radio Prop.) 269 (1960).

Taylor, W. L., Daytime attenuation rates in the VLF band using atmospherics, J. Research NBS 64D (Radio Prop.) No. 4, 349-355 (July-Aug. 1960).

Wait, James R., Terrestrial propagation of very-low-frequency radio waves, a theoretical investigation, J. Research NBS 64D (Radio Prop.) No. 2, 153-204 (March-April 1960).

Wait, James R., A new approach to the mode theory of VLF propagation, J. Research NBS 65D (Radio Prop.) No. 1, 37-46 (Jan.-Feb. 1961).

Wait, James R., and Kenneth Spies, Influence of earth curvature and the terrestrial magnetic field on VLF propagation, J. Geophys. Research 65, No. 8, 2325-2331 (Aug. 1960).

Yabroff, I. W., Reflection at a sharply bounded ionosphere, Proc. IRE 45, 750 (1957a).

Yabroff, I. W., Tech. Report No. I, July 29, 1957. R.P.L. Stanford ASTIA Doc. AD 136, 457 (1957b).

(Paper 65D5-151) 